\title{
CLASSIFIER FUSION OF HIGH-RESOLUTION OPTICAL AND SYNTHETIC APERTURE RADAR (SAR) SATELLITE IMAGERY FOR CLASSIFICATION IN URBAN AREA
}

\author{
T. Alipour Fard ${ }^{\text {a, }}$, M. Hasanlou ${ }^{a}$, H. Arefi ${ }^{\text {a }}$ \\ ${ }^{a}$ Dept. of Geomatics and Surveying Eng., University of Tehran, Tehran, Iran \\ - (tayebalipour, hasanlou, hossein.arefi)@ut.ac.ir
}

KEY WORDS: Classifier Fusion, SAR Images, Optical Images, Feature Extraction, Classification

\begin{abstract}
:
This study concerned with fusion of synthetic aperture radar and optical satellite imagery. Due to the difference in the underlying sensor technology, data from synthetic aperture radar (SAR) and optical sensors refer to different properties of the observed scene and it is believed that when they are fused together, they complement each other to improve the performance of a particular application. In this paper, two category of features are generate and six classifier fusion operators implemented and evaluated. Implementation results show significant improvement in the classification accuracy.
\end{abstract}

\section{INTRODUCTION}

These days, many remote sensing satellite sensors are acquiring information at different spatial, spectral and temporal resolutions. However, the information provided by the individual sensors might be incomplete or imprecise for a given application (Hall and Llinas, 1997). Combing microwave and optical sensors can help in discriminating the different classes since they are complementary to each other (Pohl and Van Genderen, 1998). Many studies have combined optical image and microwave image to improve mapping accuracy in different scenarios. SAR and optical imagery can be integrated in different approaches to improve the data and information content during image processing for information extraction. Classifier fusion is a technique which can combine the optical and SAR sensor data, at decision level. The purpose of radar and optical fusion is mainly to use synergy between SAR and optical images for increasing discriminant power between different classes (van der Sanden and Thomas, 2004; Schist adSolberg et al., 1994).

Optical data contains information on the reflective and emissive characteristics of the targets in terms of spectral intensity. This spectral information is related the chemical composition and moisture content of the observed target. On the other hand, SAR data contains information on the geometric structure, surface roughness and dielectric properties of natural and manmade objects. As an example, spectral signature is the information inferred from optical data, which is used to characterize ground targets. However, data provided by sensors is always subjected to some level of uncertainty and inconsistency. Data fusion algorithms reduce the uncertainty by combining data from several sources (Waleed and Alaa, 2013). Data fusion refers to combining information from two or more sources together to improve the quality and interpretability of the source data. This can be achieved at any one of the three different processing levels of the image information: pixel (low- level), feature (medium-level) and classifier (high-level) fusion approaches (Yitayew, 2012).

- Pixel level fusion is a low level fusion where different source images are combined to produce a single fused image.

- Feature level fusion is an intermediate level of fusion, which requires the merging of extracted features.

- Classifier level fusion is a high level fusion which is used to integrate separately processed image information using decision rules.

Many studies have been published to combine SAR and optical data for a number of applications (Amarsaikhan, 2007, Ricchetti, 2001, Venkataraman, 2004, Solberg, 1994, Hong, 2014). To mention some of the applications, two SAR datasets from ERS-1/2 (C-band) and JERS-1 (L-band) are fused with a multi-spectral dataset from the SPOT satellite for the purpose of urban land cover classification (Amarsaikhan, 2007). In (Ricchetti, 2001) a study was conducted to fuse images from ERS-1 satellite and Landsat thematic mapper sensors for geological study purposes. Snow cover mapping using these two different datasets was demonstrated in (Venkataraman, 2004). Another application example where SAR and optical data sets are integrated is land cover mapping, (Solberg, 1994, Hong, 2014).

However, most of this study concentrated on image fusion techniques and feature fusion and classifier fusion were not considered. Only few studies have been conducted on extracting and combining features from SAR and optical datasets for urban applications.

This stud, focus on extracting feature from SAR and optical data, performing and evaluating different classifiers (NN, $\mathrm{KNN}$,) on features and fusion of class labels for each pixel has been obtained by different classifiers. The main motivation of extracting and combining class labels of different classifiers is that specific information can be retained in the fusion process

* Corresponding author. 
and could be helpful to improve the fusion results by using different classifiers that each classifier emphasize one aspect of data and this framework can reduce uncertainty of data. Therewith, this approaches reduce dependency of results to specific sensor versus pixel fusion and feature fusion approaches, if two basic criteria: independence and diversity in the selection of classifiers be considered.

\section{STUDY AREA AND DATA SETS}

\subsection{Study area}

A study area was selected in Southern IRAN, Shiraz City (Fig. 1). The geographic coverage of this area is about $22 \mathrm{~km} \times 18$ $\mathrm{km}$. The study area is primarily semiarid and urban is dominated class in scene.

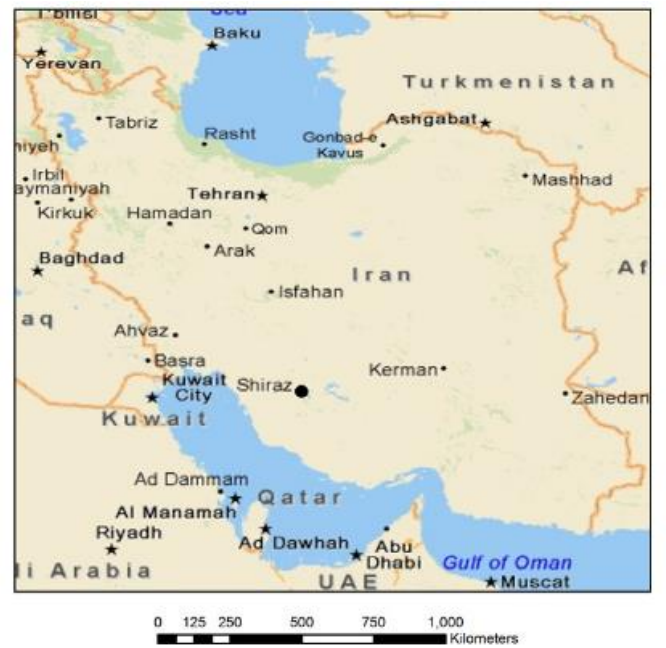

Fig. 1. Shiraz location in Iran.

\subsection{Data sets}

In this study two data sets were used. One is Synthetic Aperture RADAR (SAR) image and another is optical image.

The polarimetric SAR data is acquired in October 2009 by ALOS/PALSAR sensor was level 1.1 in Single Look Complex (SLC) format. The product contains $\mathrm{HH}, \mathrm{VV}, \mathrm{HV}$ and $\mathrm{VH}$ images and leader files. The SLC data is more advantage than detected product because phase information is preserved in the SLC data products. The Phased Array type L-band Synthetic Aperture Radar (PALSAR) is an active microwave sensor using L-band frequency to achieve cloud-free and day-and-night land observation. The pixel spacing is $5 \mathrm{~m}$ in the azimuth and $30 \mathrm{~m}$ in the range direction (Fig.2.a).

The multi-spectral dataset is acquired by the IKONOS in September 2009 and has four co-registered spectral channels. Originally, all except the 3 th band were at $4 \mathrm{~m} \times 4 \mathrm{~m}$ resolution; the panchromatic band was at a resolution of $1 \mathrm{~m} \times 1 \mathrm{~m}$. However, it is provided after all the four bands are co-registered to a common resolution of $4 \mathrm{~m} \times 4 \mathrm{~m}$ (Fig.2.b).

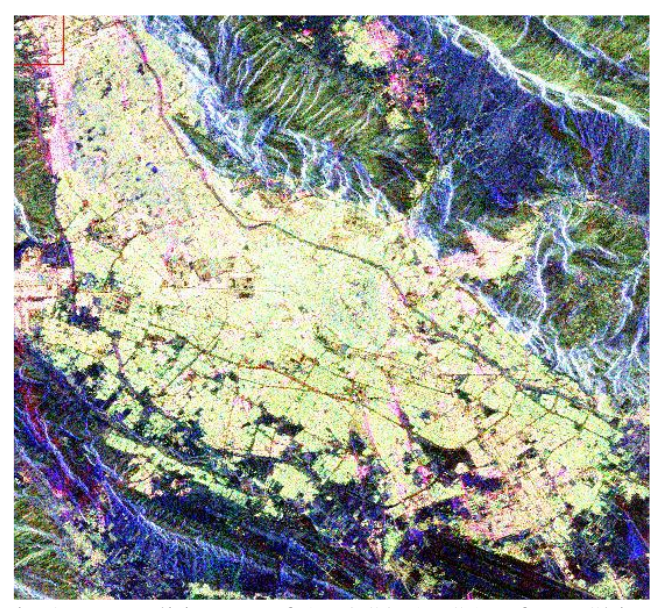

Fig.2.a : Pauli image of ALOS/PALSAR from Shiraz City, Iran

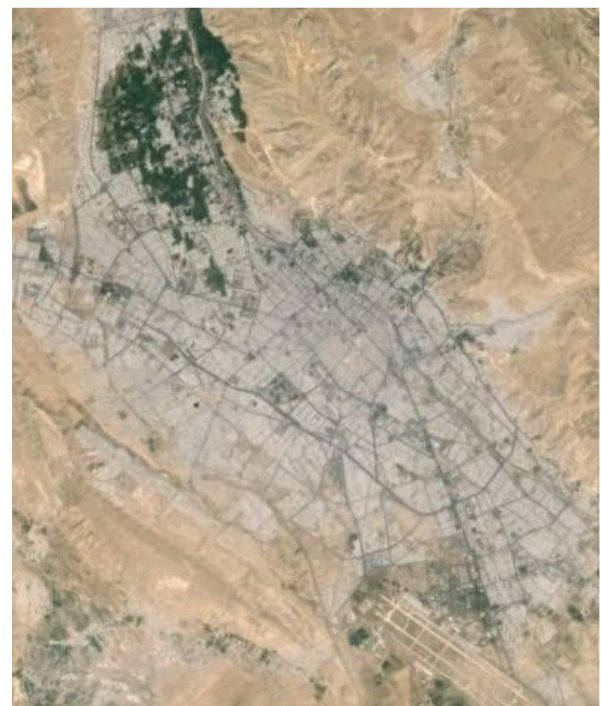

Fig.2.b : IKONOS image from Shiraz City, Iran

\subsection{Train and test data}

In this paper, an intuitive method to collect training data is used .About $2 \%$ of the pixels as training data and $15 \%$ of the pixels were selected as test data. Figure 3 shows the distribution of training data.

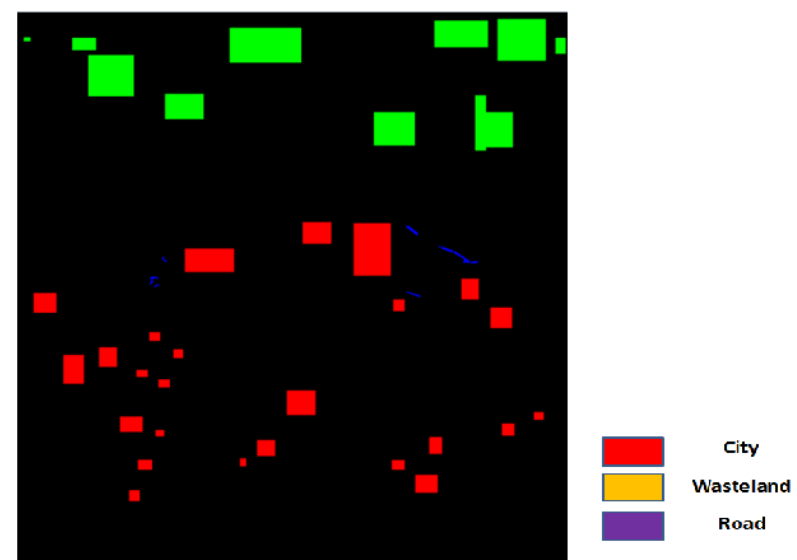

Fig.3 : Distribution of training data 


\section{METHODOLOGY}

A proper preparation of data is a very important prerequisite for a successful data fusion. Following paragraph present our approach methodologically including the data processing chain.

- $\quad$ Input image data are of multi-sensor nature:

○ Manual Co-registration

○ Resampling

- Pre-Processing

- Speckle noise reduction (for SAR image)

- Noise reduction (for optical image)

- Feature Extraction and Concatenation

- SAR image (SAR Discriminator, Target Decomposition, ...)

- Optical Image (First order, GLCM)

- Concatenating and normalization two sets of features

- Classification methods

- Neural Network

- Decision Tree

- Quadratic Classifier

- K-Nearest Neighbor(KNN)

- Fusion Scheme

- Class label combination

- Classification accuracy assessment by calculating overall accuracy and kappa coefficient.

\subsection{Speckle noise reduction}

The original image is contaminated with speckle noise caused by the coherent illumination used in SAR system sensors. Speckle is a kind of noise which decrease the quality of an image and makes interpretation more difficult and thus needs to be removed or reduced to analysis (Hong, 2014). First, the Gamma filter was used to remove the speckle existing in the SAR image; secondly, a conventional median filter was used to remove the heterogeneity existing in the image which has been de-speckled. The filtering window size is 3 by 3 pixels.

\subsection{Feature Generation}

Feature extraction refers to the process of transforming an input dataset into a new representation set of features which accurately and concisely represents the original information (Yitayew, 2012). An obvious advantage of feature extraction when assisted with feature selection is that only small amount of memory and processing time will be required in the feature space as redundant information is removed in the process.

This section is devoted to the extraction of the different features considered in this paper. In total, thirty-seven features; twentythree from each of the three PolSAR datasets and fourteen from the IKONOS dataset are extracted and used for this study.

\subsubsection{Polarimetric Features}

Polarimetric features can be generally grouped into two broad categories, the SAR discriminator is first that contains features processed from a simple transform of the original dataset (from the covariance matrix for example) and the second category contains features extracted based on the different target decomposition theorems. In this study, it is found that the twenty-three features considered have a rich distinguishing power for the different classes from both category that summarized in Table 1.

\begin{tabular}{|c|c|}
\hline Name of Feature & Formula \\
\hline $\begin{array}{l}\text { Total } \\
\text { backscattering } \\
\text { power }\end{array}$ & $S P A N=\left|S_{h h}\right|^{2}+\left|S_{v v}\right|^{2}+2\left|S_{h v}\right|^{2}$ \\
\hline Polarization ratio & $\begin{array}{l}10 \cdot \log \left(\frac{\left|S_{V V}\right|^{2}}{\left|S_{H H}\right|^{2}}\right) \\
10 \cdot \log \left(\frac{\left|S_{H V}\right|^{2}}{\left|S_{H H}\right|^{2}}\right) \\
10 \cdot \log \left(\frac{\left|S_{X V V}\right|^{2}}{\left.S_{V V}\right|^{2}}\right)\end{array}$ \\
\hline $\begin{array}{l}\text { Elements of } \\
\text { covariance matrix }\end{array}$ & $\begin{array}{c}\sigma_{C_{11}}=10 \log \left(\left|S_{h h}\right|^{2}\right), \sigma_{C_{12}}=10 \log \left(\sqrt{2} S_{h h} S_{h v}^{*}\right) \\
\sigma_{C_{13}}=10 \log \left(S_{h h} S_{v v}^{*}\right), \sigma_{C_{22}}=10 \log \left(2\left|S_{h v}\right|^{2}\right) \\
\sigma_{C_{23}}=10 \log \left(\sqrt{2} S_{h v} S_{v v}^{*}\right), \sigma_{C_{33}}=10 \log \left(\left|S_{v v}\right|^{2}\right)\end{array}$ \\
\hline $\begin{array}{l}\text { Elements of } \\
\text { coherency matrix }\end{array}$ & 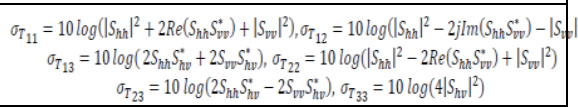 \\
\hline $\begin{array}{l}\text { Degree of } \\
\text { polarization }\end{array}$ & $D o P=\frac{\sqrt{S_{1}^{2}+S_{2}^{2}+S_{3}^{2}}}{S_{0}}$ \\
\hline $\begin{array}{l}\text { Elements } \\
\text { backscatter matix } \\
\text { / SPAN }\end{array}$ & $R_{h h}=\frac{\left|S_{h h}\right|^{2}}{S P A N}, R_{h v}=\frac{\left|S_{h v}\right|^{2}}{S P A N}, R_{v v}=\frac{\left|S_{v v}\right|^{2}}{S P A N}$ \\
\hline $\begin{array}{l}\text { Pauli } \\
\text { decomposition }\end{array}$ & $|\alpha|^{2}=\left|\frac{S_{h h}+S_{v v}}{\sqrt{2}}\right|^{2},|\beta|^{2}=\left|\frac{S_{h h}-S_{v v}}{\sqrt{2}}\right|^{2},|\gamma|^{2}=\sqrt{2} S_{h v}$ \\
\hline
\end{tabular}

Table 1. List of polarimetric features that generated in this paper

Where Shh and $\mathrm{S}_{\mathrm{vv}}$ are called co-polarized elements and Svh and Shv are called cross-polarized elements of scatter matrix.

Some of this polarimetric features are shown as images in figures 4.a-4.d.

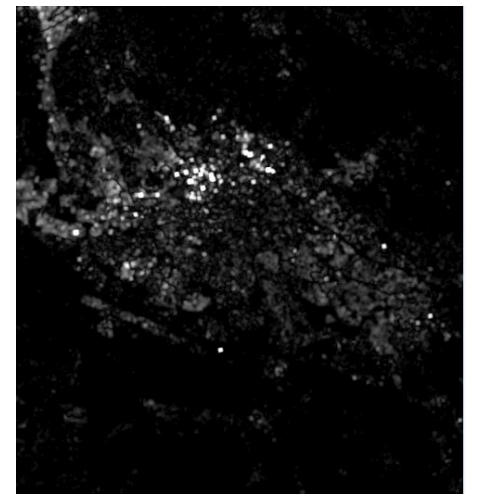

Fig. 4.a :Elements of covariance matrix (c22)

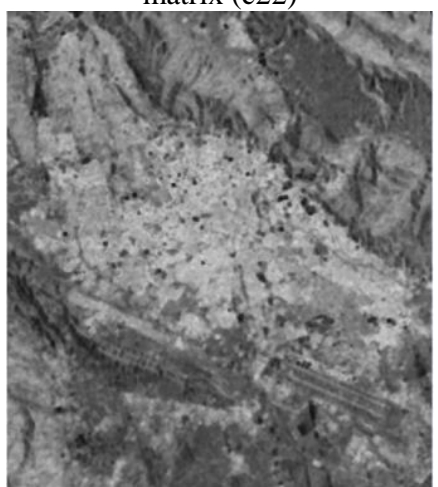

Fig. 4.c : Elements of backscatter matix / SPAN (Shh/Span)

\subsubsection{Optical Features}

There are four approaches to extraction features form very high resolution images: a) statistical b) structural c) frequency based d) model based. In this study, due to nature of urban area, statistical feature are generated. Though, among statistical 
feature GLCM textural features are well known and well describe in (Haralick, 1979). In this paper three category of textural feature that represent homogeneity, smoothness and correlation between gray levels were selected. This feature summarized in Table 2.

\begin{tabular}{|c|c|}
\hline $\begin{array}{l}\text { Name } \\
\text { feature }\end{array}$ & formula \\
\hline Contrast & $\sum_{i=0}^{N N_{g}-1 N N_{g}-1} \sum_{j=0}^{(i-j)^{2} P(i, j)}$ \\
\hline Dissimilarity & $\sum_{i=0}^{N_{g}-10 N_{g}^{-1}} \sum_{j=0}^{j}|i-j| P\left(i_{i} j\right)$ \\
\hline $\begin{array}{l}\text { Angular } \\
\text { Second } \\
\text { Moment }\end{array}$ & 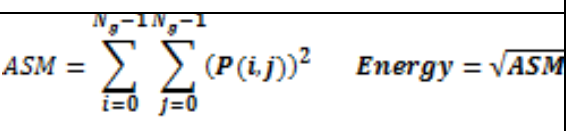 \\
\hline Entropy & $\sum_{i=0}^{N_{g}-1 N_{g}-1} \sum_{j=0}^{P(i, j)} \ln P(i, j)$ \\
\hline $\begin{array}{l}\text { Homogeneity } \\
\text { (IDM) }\end{array}$ & $\sum_{i=0}^{N_{g}-11 N_{g}-1} \sum_{j=0}^{P(i, j)} \frac{P(i-j)^{2}}{1+(i)}$ \\
\hline $\begin{array}{l}\text { Mean and } \\
\text { Variance }\end{array}$ & 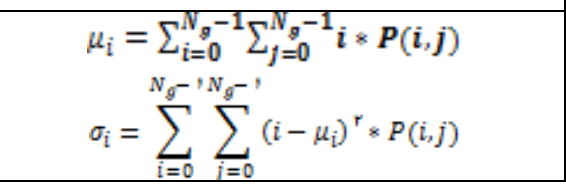 \\
\hline
\end{tabular}

Table 2. List of optical features that generated in this paper

Where:

$P(i, j)$ : number of pixels with $\mathrm{DN}=\mathrm{i}, \mathrm{DN}=\mathrm{j}$ in certain direction and distance and $\mathrm{Ng}$ is quantization level of image.

\subsection{Classifier Fusion}

Combining classifiers is one of the most widely explored methods in pattern recognition in the recent years. These techniques have been shown to reduce the error rate in classification tasks in opposite to single classifiers.

Designing a suitable method of decision combinations is a key point for the ensemble's performance. In this paper, class combination methods were used. These techniques are classified into three categories: a) abstract level b) rank level c) measurement level. Class label combination widely used that well describe in (Kuncheva, 2002, Ruta, and Gabrys, B, 2000). Because we implement majority voting, product, mean, $\min / \mathrm{max}$ and median operators selected for fusion of output labels of each classifiers.

\section{RESULTS AND DISCUSSION}

After generating feature from both datasets, normalization and concatenation done. well known classifiers include Neural Network (NN), Decision Tree(DT), K-Nearest Neighbors(KNN) ,Quadratic Discriminant Classifier (QDC) and six classifier fusion (Majority voting, Product, Median, Max, Min,Mean) on datasets were implement. Figure 5 shows the results of the implementation of classifiers without fusion and figure 6 shows results fusion methods in terms of the overall accuracy and kappa coefficient.

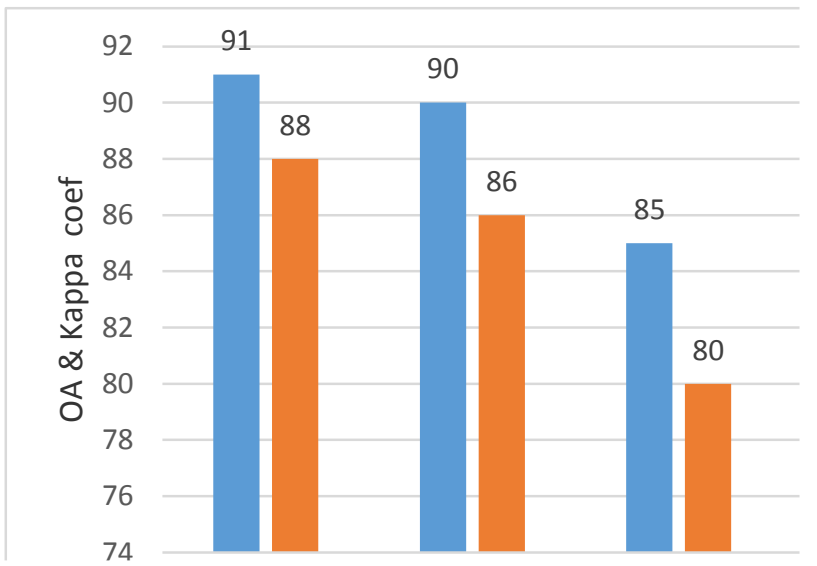

Figure 5: Results of Implementation (no fusion)

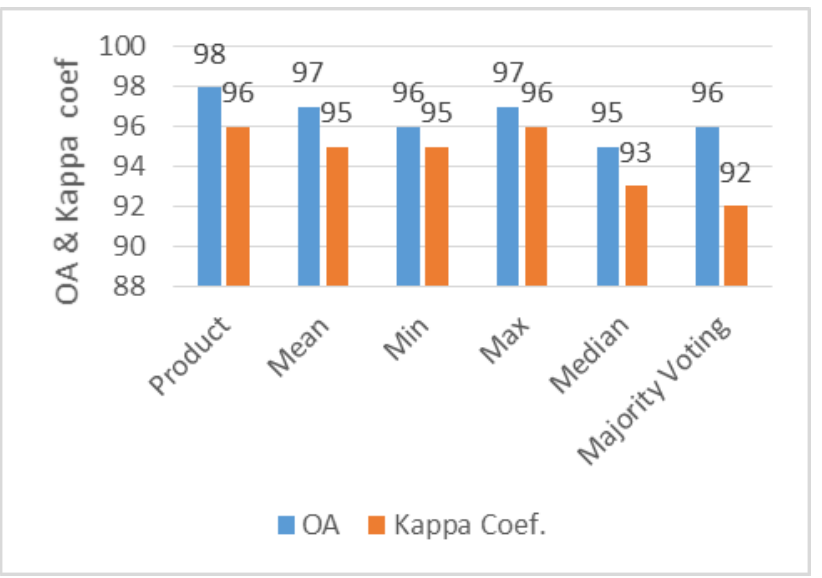

Figure 6: Results of Implementation (fusion)

Product method has high performance through the fusion of the thirty-seven features derived from the whole available dataset and can be seen in fusion methods the results have improved by 4 to 8 percent. Classifier selection is a good diversity, Therefore, the fusion operators are substantially improved classification accuracy. Because the number of classes is 3 , results of min and max operators are slightly different. The classification map shown in Figure 7 represents the best classification accuracy, which is related to product fusion operators by overall accuracy of $98 \%$.

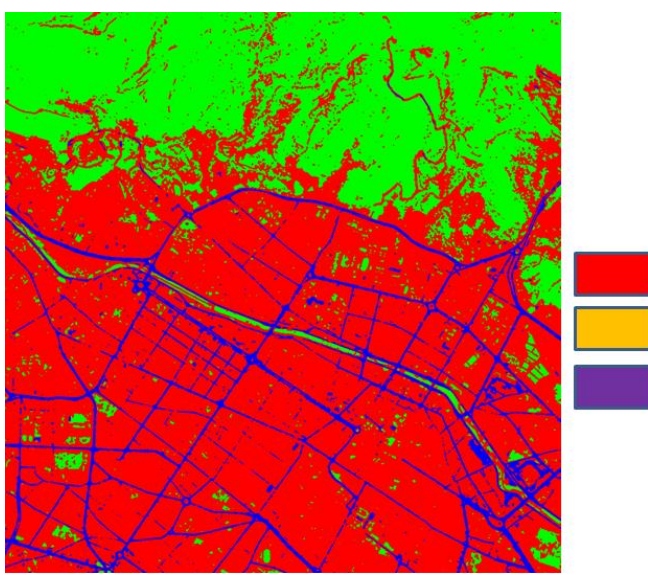

City

Wasteland

Road

Fig.7. classification map by product operator (best result, $\mathrm{OA}=98 \%$ ) 


\section{CONCLUSION}

We have classified ALOS/PALSAR and IKONOS images by classifier fusion methods.Six simple classifier fusion methods have been studied: minimum, maximum, mean, median, majority vote, and product. It was shown that product operators in this case is the best method. It was observed that, fusion methods can be used to improve classification accuracy about 4 $8 \%$.

\section{ACKNOWLEDGMENT}

The authors would like to thank Mr. Akbar Alaei for providing the ALOS/PALSAR image.

\section{REFRENCES}

Hall, D.L. and Llinas, Jl., 1997, "An introduction to multisensor data fusion”, Proceedings of the IEEE, 85(1):6-23,

Pohl, C and Van Genderen, Jl., 1998, Review article multisensor image fusion in remote sensing: concepts, methods and applications. International journal of remote sensing, 19(5), pp. 823-854.

Van der Sanden, J.J., Thomas, S.J. (Eds.), 2004. Applications Potential of Radarsat-2Supplement One. Canada Centre of Remote Sensing, Ottawa, ON, p. 135.

Schistad-Solberg, A., Jain, A., Taxt, T., 1994. Multisource classification of remotely sensed data: fusion of Landsat TM and SAR images. IEEE Transactions on Geo-science and Remote Sensing, 32, pp.768-776.

Waleed A. and Alaa Kh., 2013, Handling Data Uncertainty and Inconsistency Using Multisensor Data Fusion, Advances in Artificial Intelligence, Research article, volume 2013, pp.1-11.

Yitayew, T.G., 2012, Multi-sensor Data Fusion and Feature Extraction for Forest Applications, Master's Thesis in Physics, University of Troms, pp.7-29.

Amarsaikhan, D., Ganzorig, M., Ache, P., and Blotevogel, H.,2007, The integrated use of optical and InSAR data for urban land-cover mapping. International Journal of Remote Sensing, 28(6):1161-1171

Ricchetti, E., 2001, Visible infrared and radar imagery fusion for geological application: A new approach using DEM and sun-illumination model. International Journal of Remote Sensing, 22(11):2219-2230.

Venkataraman, G., Mahato, B.C., Ravi, S., Rao, YS, Mathur, P. et al., 2004, Fusion of optical and microwave remote sensing data for snow cover mapping. In Geoscience and Remote Sensing Symposium, 2004. IGARSS'04. Proceedings. volume 4, pages 2554-2557. IEEE, 2004.

Solberg, A.H.S., Jain, A.K., and Taxt, T., 1994, Multisource classification of remotely sensed data: fusion of Landsat TM and SAR images. Geoscience and Remote Sensing, IEEE Transactions on, 32(4):768-778.

Hong, G., Zhang, A., Zhou, F., Brisco, B., 2014, Integration of optical and synthetic aperture radar (SAR) images to differentiate grassland and alfalfa in Prairie area, International
Journal of Applied Earth Observation and Geoinformation, 28, pp. 12-19.

Haralick, R.M., 1979, Statistical and structural approaches to texture, Proc IEEE, vol. 67, pp. 786-804, 1979.

Kuncheva, L., 2002, A Theoretical Study on Six Classifier Fusion Strategies, IEEE Transaction on Pattern Analysis and Machine Intelligence, VOL. 24, NO. 2, pp.281-286.

Ruta, D., Gabrys, B., 2000, An Overview of Classifier Fusion Methods, Computing and Information Systems, 7 pp.1-10. 